\title{
Simultaneous uvby photometry of the $\delta$ Sct star HD 129231
}

\author{
E. Rodríguez ${ }^{1}$, M. J. López-González ${ }^{1}$, A. Rolland ${ }^{1}$, V. Costa ${ }^{1}$, and S. F. González-Bedolla ${ }^{2, \star}$ \\ 1 Instituto de Astrofísica de Andalucía, CSIC, PO Box 3004, 18080 Granada, Spain \\ 2 Instituto de Astronomía, UNAM, PO Box 70-264, CP-4510 México D.F., México
}

Received 7 May 2001 / Accepted 26 June 2001

\begin{abstract}
We present the results of a three-year Strömgren uvby photometric study of the recently discovered multiperiodic low amplitude $\delta$ Sct-type pulsator HD 129231. Some additional $\mathrm{H}_{\beta}$-Crawford measurements were also collected. Multiperiodicity is needed to describe the pulsational behaviour of this variable during each of the observing runs. A set of five significant frequencies has been found as the best fitting for the 1997 dataset, but only the two main ones result as significant during the 1995 and 1996 observing runs. Amplitude variations from season to season are also found for the main frequency $f_{1}$. Nonradial pulsation is suggested for some of the modes. Using the derived uvby $\beta$ indices, the most relevant physical parameters of HD 129231 are also determined placing this object as a hot Population I $\delta$ Sct star evolving on its main sequence stage.
\end{abstract}

Key words. stars: variables: $\delta$ Scu - stars: individual: HD 129231 - stars: oscillations - techniques: photometric

\section{Introduction}

Long-term monitoring of high quality photometry on stars in the lower part of the classical Instability Strip reveals the existence of more variables in this part of the H-R diagram and constantly increases the number of known $\delta$ Sct-type pulsators. A large number of these long-term monitoring projects are the natural consequence of observations of $\mathrm{A}-\mathrm{F}$ stars used as comparison or check stars for observations of already known variables. This is the case of the low amplitude $\delta$ Sct-type variables HD 130484 and HD 129231 (Rodríguez et al. 1999a, b) which were used as check stars during an observational program carried out on the medium amplitude $\delta$ Sct star IP Vir $\left(V=11^{\mathrm{m}} 60\right.$, $\Delta V=0$. $10, P=0$ d 0652 , Rodríguez et al. 2000). In both cases, observations were also collected by the Hipparcos satellite (ESA 1997), but no variability was detected because of the low $S / N$ ratio reported as compared to the small amplitudes of photometric light variations presented by these two objects.

In a preliminary analysis, the multiperiodic behaviour of HD 129231 was evident with a main period of about 46 min and a peak to peak amplitude of 0.01 in the Johnson $V$ band, based on observations collected through the years 1995 and 1996. In the present work, we carefully reanalysed the 1995 and 1996 datasets together with a new and much larger dataset collected during the spring of 1997. The observations are described in Sect. 2; a detailed frequency analysis is performed in Sect. 3 and the

Send offprint requests to: E. Rodríguez, e-mail: eloy@iaa.es * Deceased. most relevant astrophysical parameters of this star are derived in Sect. 4. Section 5 summarizes the main results.

\section{Observations}

The observations were carried out during the springs of 1995 and 1996 at the observatories of Sierra Nevada, Spain (SNO, $90 \mathrm{~cm}$ telescope) and San Pedro Mártir, Mexico (SPMO, $1.5 \mathrm{~m}$ telescope). Both telescopes are equipped with identical six-channel uvby $\beta$ spectrograph photometers for simultaneous measurements in uvby or the narrow and wide $\mathrm{H}_{\beta}$ channels (Nielsen 1983).

The main object for this study was the $\delta$ Sct variable IP Vir with HD 129231 as one of the check stars, but the observations revealed the latter as another $\delta$ Scttype variable with low amplitude variations and short period (Rodríguez et al. 1999b). Thus, we decided to collect new measurements for this variable during the spring of 1997 at SNO. Table 1 lists the journal of uvby observations collected for HD 129231. As it can be seen, the dataset collected in 1997 is much larger than the obtained in previous years. Additionally, a few measurements were collected some nights in $\mathrm{H}_{\beta}$. The other check star used during the 1995 and 1996 observing runs was HD 130484 which resulted as another $\delta$ Sct variable with also very low amplitude, but longer period $\left(\sim 3^{\mathrm{h}}\right)$ (Rodríguez et al. 1999a).

During the observations carried out in 1995 and 1996 , IP Vir $\left(V=11^{\mathrm{m}} 60, \mathrm{~F} 1\right)$ was considered as the variable whereas $\mathrm{C} 1=\mathrm{HD} 129727(V=9 \mathrm{~m} 5$, F0) was used as the main comparison star with E1 = HD 129231 
Table 1. Journal of uvby observations.

\begin{tabular}{lccr}
\hline Year & Observatory & Nights & Points \\
\hline 1995 & SNO & 4 & 166 \\
& SPMO & 2 & 17 \\
1996 & SNO & 4 & 241 \\
& SPMO & 9 & 187 \\
1997 & SNO & 21 & 1473 \\
\hline
\end{tabular}

Table 2. uvby $\beta$ indices obtained for E1, E2 and comparison stars. The pairs below the star names are the number of points collected for each object in uvby and $\beta$, respectively.

\begin{tabular}{crrrrr}
\hline Object & $V$ & $b-y$ & $m_{1}$ & $c_{1}$ & \multicolumn{1}{c}{$\beta$} \\
\hline $\mathrm{E} 1=$ HD 129231 & 7.834 & 0.062 & 0.183 & 1.007 & 2.885 \\
$(2084,30)$ & 6 & 3 & 4 & 8 & 7 \\
$\mathrm{E} 2=$ HD 130484 & 8.786 & 0.188 & 0.172 & 0.946 & 2.771 \\
$(454,9)$ & 9 & 4 & 3 & 5 & 12 \\
$\mathrm{C} 1=$ HD 129727 & 9.480 & 0.261 & 0.143 & 0.515 & 2.693 \\
$(339,16)$ & 9 & 4 & 4 & 7 & 11 \\
$\mathrm{C} 2=$ HD 130256 & 6.623 & 0.024 & 0.136 & 0.926 & 2.849 \\
$(1062,11)$ & 5 & 3 & 3 & 7 & 7 \\
$\mathrm{C} 3=$ HD 129584 & 7.330 & 0.017 & 0.154 & 1.019 & 2.906 \\
$(371,6)$ & 6 & 3 & 4 & 7 & 6 \\
\hline
\end{tabular}

$\left(V=7^{\mathrm{m}} 8, \mathrm{~A} 2\right)$ and $\mathrm{E} 2=\mathrm{HD} 130484\left(V=8^{\mathrm{m}} 8, \mathrm{~F} 2\right)$ as check stars. The sequence was, generally, C1, E1, IP Vir, $\mathrm{C} 1, \mathrm{E} 2$, IP Vir during the first nights. Once the variability in E1 and E2 was suspected, the sequence was changed to C1, E1, E2, IP Vir. Sky measurements were made every 2 or 3 cycles. 611 uvby data were collected for E1, 454 for E2 and about 300 for C1. Each integration consisted of $50 \mathrm{~s}$ for $\mathrm{C} 1$ and E2 and $40 \mathrm{~s}$ for E1. This means an internal error per observation better than $0 .{ }^{\mathrm{m}} 006$ for any of the stars and filters. The extinction corrections were based on nightly coefficients determined from the comparison star. Then, magnitude differences of each object relative to $\mathrm{C} 1$ were calculated by means of linear interpolation.

With the sake of getting better precision in the resulting magnitude differences, two new comparison stars were used during the 1997 observing run. In this case, C2 = HD 130256 ( $V=6{ }^{\mathrm{m}} 6$, A0) was used as main comparison star and $\mathrm{C} 3=\mathrm{HD} 129584\left(V=7^{\mathrm{m}} \cdot 3, \mathrm{~A} 0\right)$ as check star. C1 was also used during one night with two purposes, first, to verify the constancy of this object and second, to make compatible the magnitude differences relative to $\mathrm{C} 1$ with those relative to $\mathrm{C} 2$. Now, the internal error per observation is of about $0 .{ }^{\mathrm{m}} 002$ in the $u$ filter for C3, namely the worst case.

During the observations reported here, none of the comparison stars showed any sign of variability. When considering the 1997 dataset, each night, the standard deviations for $\mathrm{C} 3-\mathrm{C} 2$ differences were always better than 0. $007,0.004,0.004$ and 0.005 for $u, v, b$ and $y$, respectively. In addition, the mean values obtained for the C3-C2 differences on each of the nights were always the same within 0.002 , as standard deviation, for any of the filters. Moreover, when we consider the full sample, the standard deviations of $\mathrm{C} 3-\mathrm{C} 2$ were found to be of $0.0060,0.0037,0.0036$ and $0 .^{\mathrm{m}} 0040$ for $u, v, b$ and $y$, respectively.

Furthermore, a frequency analysis was performed to our data in order to investigate the existence of possible small variabilities in $\mathrm{C} 3$ or $\mathrm{C} 2$. When a Fourier analysis was applied (using the method described in Rodríguez et al. 1998) to the $v$ filter, we obtained C3 and C2 not showing any sign of variability with amplitude larger than about 0.0009 , in the range from 0 to $50 \mathrm{~cd}^{-1}$. Similar results were obtained for the other two $b$ and $y$ filters. Hence, C3 and C2 can be considered as constant stars within the observational errors. The same seems to be true for C1. This case, the limit in amplitude can be established around 0 . 0015 when we compare the results from the frequency analyses for E1-C1 and E2-C1 using the 1995 and 1996 datasets.

In order to transform our data into the standard $u v b y \beta$ system, we have used the same procedure described in Rodríguez et al. (1997a). The data obtained, as magnitude differences, variable minus $\mathrm{C} 2$, in the standard system versus Heliocentric Julian Day, have been deposited in the Commission 27 IAU Archives of Unpublished Observations, file $346 \mathrm{E}$, and can also be requested from the authors. Derived standard $u v b y \beta$ indices together with the standard deviations and number of points collected in uvby and $\beta$ for each object are listed in Table 2 . The results concerning IP Vir are not listed because they will be the subject of another study. Our derived Strömgren values are in very good agreement with those found in the bibliography (Hauck \& Mermilliod 1998) for C1 and E1 (the only ones with available uvby $\beta$ photometry from earlier authors). The $V$ values are also in very good agreement with those listed in the Hipparcos catalogue (ESA 1997) $\left(V=7^{\mathrm{m}} \cdot 83,8^{\mathrm{m}} \cdot 78,6.62\right.$ and $7^{\mathrm{m}} \cdot 32$ for $\mathrm{E} 1, \mathrm{E} 2, \mathrm{C} 2$ and C3, respectively) or Simbad Database (Simbad 2001) $\left(V=9{ }^{\mathrm{m}} 48\right.$ for $\left.\mathrm{C} 1\right)$. This is also true for the for $b-y$ values (transformed to $B-V$ by means of the Penprase's (1992) equations) as compared with those $B-V$ values derived from the measurements collected by the Hipparcos satellite (ESA 1997).

\section{Frequency analysis}

The frequency analyses were carried out using the method described in Rodríguez et al. (1998) where singlefrequency and multiple-frequency techniques are combined using both Fourier and multiple least squares algorithms. The multiple-frequency least squares technique does not depend on successive prewhitenings but calculates the best fit to the data with all considered frequencies simultaneously by minimizing the residuals. Nevertheless, prewhitening is required for purposes of presentation in order to show the low amplitude modes, and various power spectra are commonly presented as a series of panels. Experience with other previous pulsating stars shows that this program yields identical results as using the PERIOD98 (Breger 1990; Sperl 1998) package. Both 


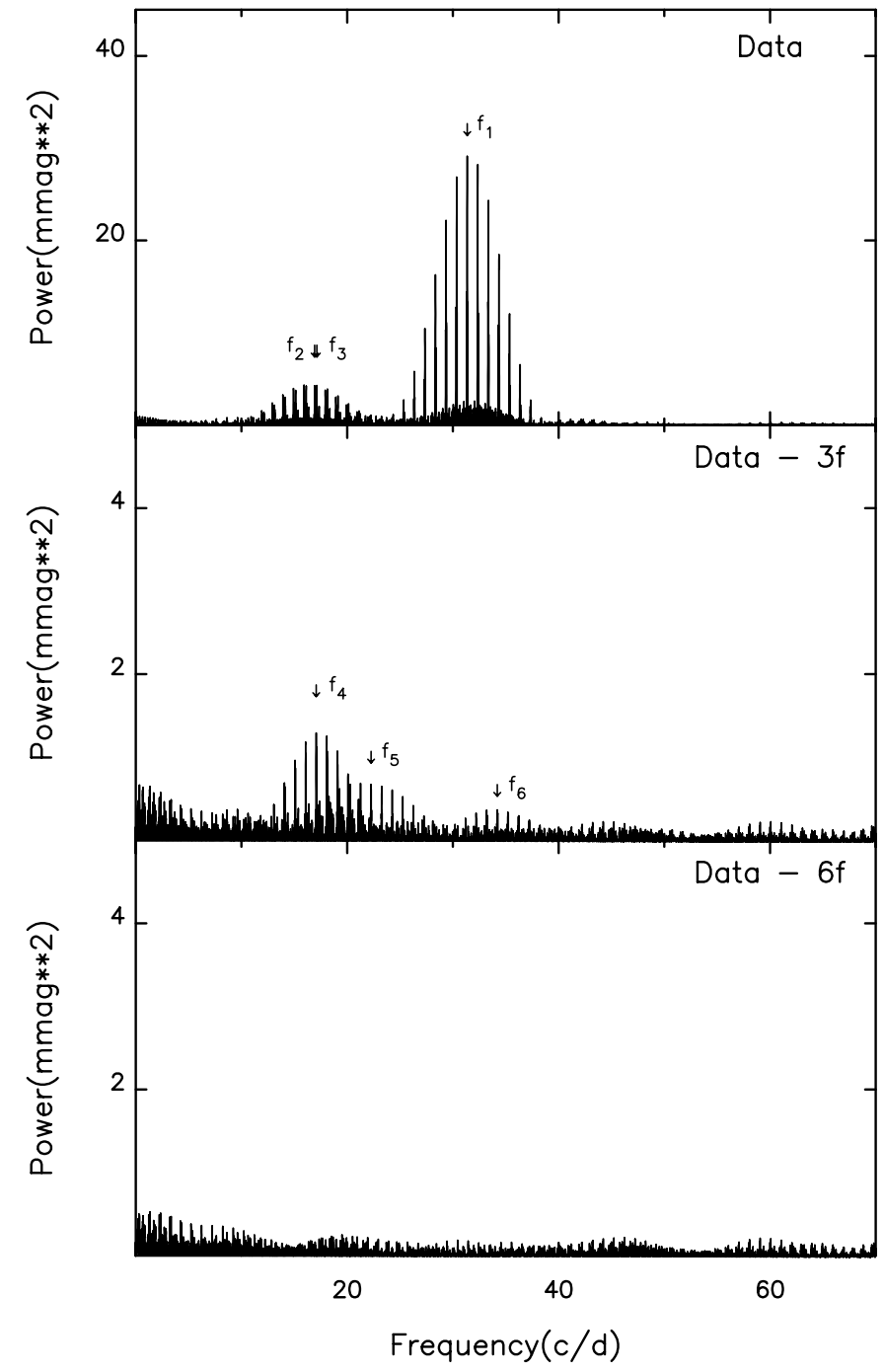

Fig. 1. Power spectra of HD 129231 in the Strömgren $v$ band corresponding to the data collected in the year 1997 and the results after removing three and six simultaneously optimized peaks.

Table 3. Frequencies, amplitudes and ratios of amplitude signal/noise obtained for the 1997 dataset and combined vby filters.

\begin{tabular}{ccr}
\hline $\begin{array}{c}\text { Frequency } \\
\left(\mathrm{cd}^{-1}\right)\end{array}$ & $\begin{array}{c}\text { Amplitude } \\
(\mathrm{mmag})\end{array}$ & $S / N$ \\
\hline$f_{1}=31.3548$ & 5.24 & 37.4 \\
$f_{2}=16.9229$ & 1.98 & 11.6 \\
$f_{3}=17.1296$ & 1.84 & 10.8 \\
$f_{4}=17.0673$ & 1.20 & 7.1 \\
$f_{5}=22.2602$ & 0.71 & 4.2 \\
$f_{6}=34.1984$ & 0.45 & 3.0 \\
\hline
\end{tabular}

programs use the same Discrete Fourier Transform (Deeming 1975).

A very important aspect in analysing the frequency content of a multiperiodic pulsator consists in judging whether or not a peak is significant in the power spectra. For this, the criterion given by Breger et al. (1993) has been followed and one peak is assumed as intrinsic of the variable when the amplitude signal/noise $(S / N)$ ratio is $\geq 4.0$. This criterion can be relaxed to 3.5 for peaks at harmonics or combination frequencies (Breger et al. 1999). In our case, the noise level is calculated by averaging the amplitudes (oversampled by a factor of 20) over $5 \mathrm{~cd}^{-1}$ regions centered around the frequency under consideration.

The dataset collected in the year 1997 was the first one analysed because of being the best (21 nights, 1473 points and the main comparison star being brighter) and hence a more detailed analysis can be performed. Next, the corresponding results can be compared with those obtained from both 1995 and 1996 datasets. The $v$ and $b$ bands were firstly analysed because the intensity in luminosity, and hence precision, is much better in these two bands. The results using the filter $v$ are shown in Fig. 1 where six frequencies appeared as significant at $f_{1}=31.355$, $f_{2}=16.923, f_{3}=17.129, f_{4}=17.069, f_{5}=22.262$ and $f_{6}=34.199 \mathrm{~cd}^{-1} \approx f_{3}+f_{4}$. The $S / N$ ratio is greater than 4.0 for all the five frequencies $f_{1}$ to $f_{5}$ whereas $S / N=3.6$ for $f_{6}$. Similar results are obtained when the $b$ filter is analysed and the same set of frequencies is found, but here $S / N=2.8$ for $f_{6}$. In the case of the $y$ filter, $f_{5}(S / N=1.5)$ and $f_{6}(S / N=2.5)$ are not significant and much noisier are the data collected in the $u$ filter.

Differences in the frequencies obtained and the significance status (different $S / N$ values in different filters for the same frequency) may be due to unreliability in the results or differences in the noise levels corresponding to different filters. In order to decide which set of frequencies is best, the $v b y$ data were combined and a new "filter" was built with the sake of getting better precision. With this new "filter" in mind, the limits of $S / N, 4.0$ for the identification of general frequencies and 3.5 for harmonics or combination frequencies, were again assumed. Filter $u$ was not considered because of being the noisiest and phase shifted with the other three. With this purpose, the $v, b$ and $y$ were aligned. Firstly, the data in each filter were normalized to zero by subtracting the averages obtained from the corresponding full datasets. Then, the $b$ and $y$ measurements were scaled to transform to $v$ amplitudes. The transformation factor, in each filter, was calculated with weighting according to the amplitudes of the three main frequencies. In our case, these factors were of 1.075 and 1.146 for transformation from $b$ and $y$, respectively. Next, the vby measurements obtained at each instant were averaged with weighting according to the precision in each filter (that is, squares of the internal errors per observation). Here, the weights were of $1.0,1.147$ and 0.563 for $v, b$ and $y$, respectively. In the case of filter $u$, the corresponding weight should be of 0.184 , but it is not used in our calculations as mentioned above.

Thus, the resulting combined vby filter was analysed and a similar set of frequencies was found. The results are shown in Table 3 together with the amplitudes and $S / N$ ratios. However, now the frequency $f_{6}$ seems to be not significant $(S / N=3.0)$. Hence, only a set with the first five $f_{1}$ to $f_{5}$ frequencies is considered and they were simultaneously optimized by means of nonlinear least squares 
Table 4. Results from the Fourier analysis applied to the dataset collected in 1997 and the four uvby filters. The corresponding signal/noise $(S / N)$ ratios are also listed.

\begin{tabular}{|c|c|c|c|c|c|c|c|c|c|c|c|c|}
\hline & \multicolumn{3}{|c|}{$u$} & \multicolumn{3}{|c|}{$v$} & \multicolumn{3}{|c|}{$b$} & \multicolumn{3}{|c|}{$y$} \\
\hline $\begin{array}{l}\text { Frequency } \\
\left(\mathrm{cd}^{-1}\right)\end{array}$ & $\begin{array}{c}A \\
(\mathrm{mag})\end{array}$ & $\begin{array}{c}\varphi \\
(\mathrm{rad})\end{array}$ & $S / N$ & $\begin{array}{c}A \\
(\mathrm{mag})\end{array}$ & $\begin{array}{c}\varphi \\
\text { (rad) }\end{array}$ & $S / N$ & $\begin{array}{c}A \\
(\mathrm{mag})\end{array}$ & $\begin{array}{c}\varphi \\
(\mathrm{rad})\end{array}$ & $S / N$ & $\begin{array}{c}A \\
(\mathrm{mag})\end{array}$ & $\begin{array}{c}\varphi \\
(\mathrm{rad})\end{array}$ & $S / N$ \\
\hline$f_{1}=31.3548$ & 0.00608 & 5.378 & 17.4 & 0.00531 & 5.279 & 35.4 & 0.00493 & 5.312 & 35.2 & 0.00435 & 5.352 & 17.4 \\
\hline 2 & 22 & 37 & & 11 & 21 & & 10 & 21 & & 13 & 30 & \\
\hline$f_{2}=16.9229$ & 0.00195 & 0.927 & 5.0 & 0.00199 & 0.853 & 10.0 & 0.00180 & 0.912 & 10.6 & 0.00177 & 0.846 & 7.7 \\
\hline 7 & 22 & 116 & & 11 & 55 & & 10 & 58 & & 13 & 75 & \\
\hline$f_{3}=17.1296$ & 0.00151 & 5.354 & 3.9 & 0.00173 & 5.114 & 8.7 & 0.00168 & 5.126 & 9.9 & 0.00182 & 5.212 & 7.9 \\
\hline 8 & 22 & 151 & & 11 & 64 & & 10 & 62 & & 13 & 73 & \\
\hline$f_{4}=17.0673$ & 0.00142 & 5.367 & 3.6 & 0.00121 & 5.195 & 6.1 & 0.00118 & 5.211 & 6.9 & 0.00096 & 5.335 & 4.2 \\
\hline 12 & 22 & 160 & & 11 & 92 & & 10 & 89 & & 13 & 139 & \\
\hline$f_{5}=22.2602$ & 0.00095 & 5.781 & 2.8 & 0.00091 & 5.541 & 5.1 & 0.00067 & 5.655 & 4.5 & 0.00037 & 5.375 & 1.7 \\
\hline 18 & 22 & 237 & & 11 & 121 & & 10 & 154 & & 13 & 355 & \\
\hline mean value & 1.4995 & & & 1.3338 & & & 1.2489 & & & 1.2110 & & \\
\hline (mag) & 2 & & & 1 & & & 1 & & & 1 & & \\
\hline $\begin{array}{r}\text { residuals } \\
\text { (mag) }\end{array}$ & 0.0061 & & & 0.0030 & & & 0.0028 & & & 0.0036 & & \\
\hline$T_{\text {or }}($ HJD $)$ & 2450485.6033 & & & & & & & & & & & \\
\hline
\end{tabular}

Table 5. Same as Table 4 for the data collected in 1996.

\begin{tabular}{|c|c|c|c|c|c|c|c|c|c|c|c|c|}
\hline & \multicolumn{3}{|c|}{$u$} & \multicolumn{3}{|c|}{$v$} & \multicolumn{3}{|c|}{$b$} & \multicolumn{3}{|c|}{$y$} \\
\hline $\begin{array}{c}\text { Frequency } \\
\left(\mathrm{cd}^{-1}\right)\end{array}$ & $\begin{array}{c}A \\
(\mathrm{mag})\end{array}$ & $\begin{array}{c}\varphi \\
(\mathrm{rad})\end{array}$ & $S / N$ & $\begin{array}{c}A \\
(\mathrm{mag})\end{array}$ & $\begin{array}{c}\varphi \\
(\mathrm{rad})\end{array}$ & $\overline{S / N}$ & $\begin{array}{c}A \\
(\mathrm{mag})\end{array}$ & $\begin{array}{c}\varphi \\
(\mathrm{rad})\end{array}$ & $S / N$ & $\begin{array}{c}A \\
(\mathrm{mag})\end{array}$ & $\begin{array}{c}\varphi \\
(\mathrm{rad})\end{array}$ & $S / N$ \\
\hline$f_{1}=31.3553$ & 0.00723 & 0.645 & 9.9 & 0.00629 & 0.612 & 23.3 & 0.00540 & 0.611 & 21.6 & 0.00484 & 0.609 & 13.8 \\
\hline 15 & 50 & 70 & & 22 & 35 & & 20 & 38 & & 29 & 61 & \\
\hline$f_{2}=16.9273$ & 0.00183 & 5.390 & 2.2 & 0.00223 & 5.186 & 5.6 & 0.00189 & 5.256 & 5.3 & 0.00189 & 5.276 & 2.8 \\
\hline 35 & 50 & 276 & & 22 & 98 & & 20 & 108 & & 29 & 157 & \\
\hline mean value & 1.4992 & & & 1.3334 & & & 1.2485 & & & 1.2105 & & \\
\hline (mag) & 4 & & & 2 & & & 1 & & & 2 & & \\
\hline $\begin{array}{r}\text { residuals } \\
\text { (mag) }\end{array}$ & 0.0073 & & & 0.0032 & & & 0.0030 & & & 0.0043 & & \\
\hline$T_{\mathrm{or}}$ (HJD) & 2450130.5810 & & & & & & & & & & & \\
\hline
\end{tabular}

fitting. The results for the frequencies and errors are listed in the first column of Table 4. This set of frequencies was then considered as fixed and the fitting for each of the four uvby filters was derived. The results are listed in the same table. Note that the listed formal errorbars in frequencies, amplitudes and phases which are obtained from the fittings are very similar to those we can obtain using the formulae from Montgomery \& O'Donoghue (1999). The $S / N$ values suggest that the frequencies $f_{1}$ to $f_{4}$ are fully confident, but the reliability of $f_{5}$ must be regarded as uncertain. Figure 2 shows the observed light variations in the $v$ band together with the solution from the Fourier fitting as applied to 1997 dataset. As can be seen, the synthetic light curves reproduce the data quite well.

The same analysis was performed for the 1995 and 1996 datasets. We applied the same procedure to combine the vby filters. Both cases, only the two main frequencies were shown to be significant. In the case of the 1996 dataset, $f_{2}$ was found to be 15.9206 , that is, $1 \mathrm{~cd}^{-1}$ alias of $16.92 \mathrm{~cd}^{-1}$. The fact of only two frequencies being significant may be due, at least in part, to the poorer quality of the data collected in 1995 and 1996 because the comparison star being fainter and the dataset being shorter. Nevertheless, the other remaining peaks from the 1997 analysis were also investigated with non-satisfactory results. The peaks corresponding to the frequencies $f_{3}$ to $f_{5}$ are not present or have undetectable amplitudes during the 1995 and 1996 observing runs. The results of the Fourier fitting for both datasets are displayed in Tables 5 and 6 and Fig. 3 .

Tables 4 to 6 show the amplitude of $f_{2}$ being the same through the three years within the observational errors, but an increase in the amplitude of $f_{1}$ occurs in 1996 decreasing again in 1997 to the same level of 1995 . Relative to the phase shifts between different bands, no definitive conclusions can be drawn because the error sizes are too large. Only $f_{1}$ seems to show reliable results in the sense that nonradial pulsation is suggested (the light maxima in the vby bands occurring earlier at longer wavelengths) (Garrido et al. 1990; Garrido 2000). The following phase shifts are obtained for $f_{1}$ and 1997 dataset: $\phi_{v}-\phi_{y}=-4.2 \pm 2.9$ and $\phi_{b}-\phi_{y}=-2.3 \pm 2.9$. 


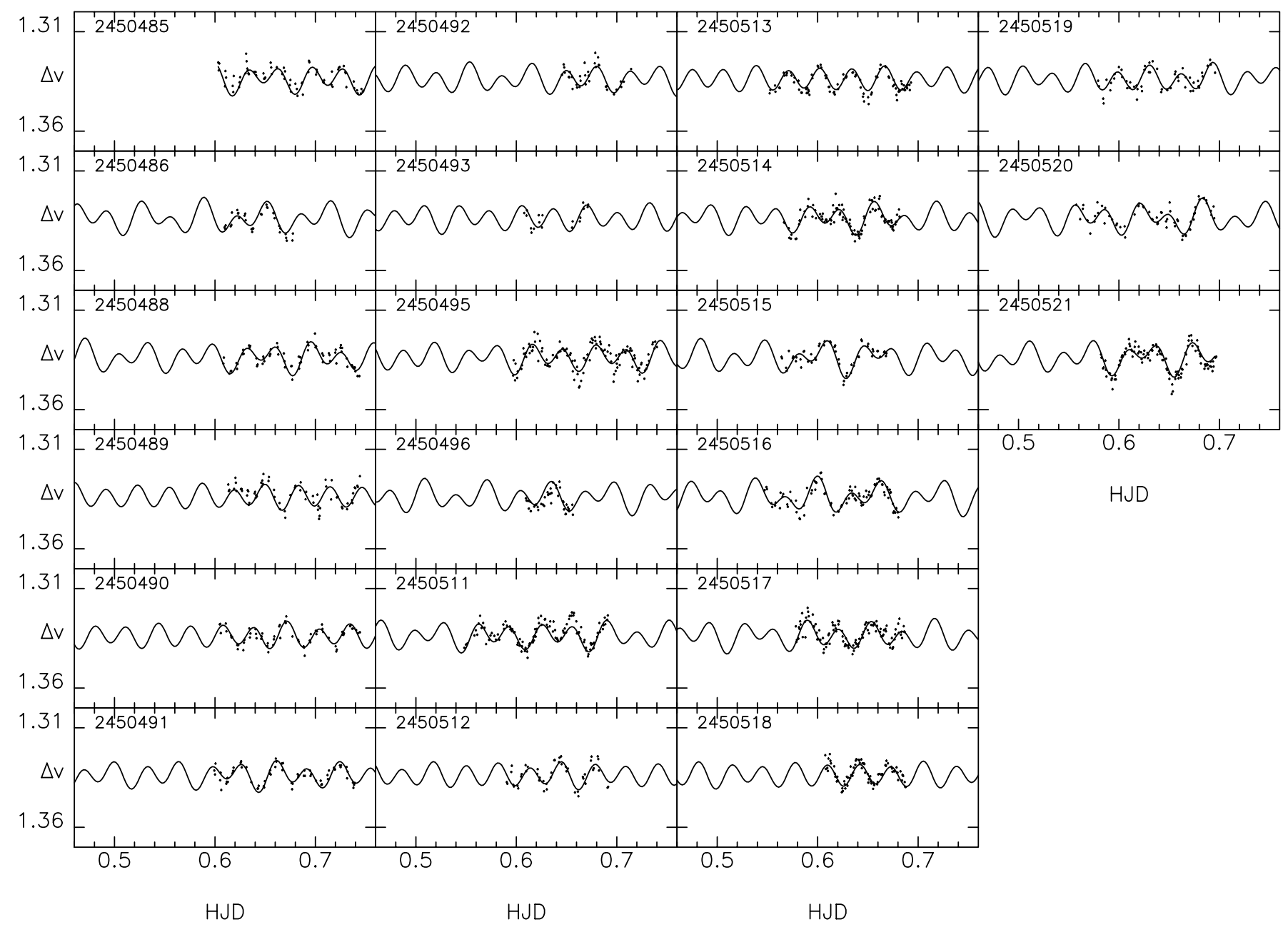

Fig. 2. Observed light variations of HD 129231 in the Strömgren $v$ band together with the corresponding Fourier fitting for the 1997 dataset.

Table 6. Same as Table 4 for the data collected in 1995 .

\begin{tabular}{|c|c|c|c|c|c|c|c|c|c|c|c|c|}
\hline & \multicolumn{3}{|c|}{$u$} & \multicolumn{3}{|c|}{$v$} & \multicolumn{3}{|c|}{$b$} & \multicolumn{3}{|c|}{$y$} \\
\hline $\begin{array}{c}\text { Frequency } \\
\quad\left(\mathrm{cd}^{-1}\right)\end{array}$ & $\begin{array}{c}A \\
(\mathrm{mag})\end{array}$ & $\begin{array}{c}\varphi \\
(\mathrm{rad})\end{array}$ & $S / N$ & $\begin{array}{c}A \\
(\mathrm{mag})\end{array}$ & $\begin{array}{c}\varphi \\
(\mathrm{rad})\end{array}$ & $S / N$ & $\begin{array}{c}A \\
(\mathrm{mag})\end{array}$ & $\begin{array}{c}\varphi \\
\text { (rad) }\end{array}$ & $S / N$ & $\begin{array}{c}A \\
(\mathrm{mag})\end{array}$ & $\begin{array}{c}\varphi \\
\text { (rad) }\end{array}$ & $S / N$ \\
\hline$f_{1}=31.3599$ & 0.00604 & 4.817 & 8.6 & 0.00493 & 4.709 & 12.3 & 0.00463 & 4.719 & 11.6 & 0.00476 & 4.743 & 10.6 \\
\hline 53 & 82 & 137 & & 37 & 77 & & 36 & 78 & & 46 & 98 & \\
\hline$f_{2}=15.9206$ & 0.00211 & 1.076 & 1.8 & 0.00236 & 1.067 & 5.2 & 0.00192 & 1.063 & 4.3 & 0.00172 & 1.107 & 2.9 \\
\hline 126 & 82 & 391 & & 37 & 160 & & 36 & 188 & & 46 & 269 & \\
\hline mean value & 1.4991 & & & 1.3335 & & & 1.2482 & & & 1.2105 & & \\
\hline (mag) & 6 & & & 3 & & & 3 & & & 3 & & \\
\hline $\begin{array}{r}\text { residuals } \\
\text { (mag) }\end{array}$ & 0.0078 & & & 0.0036 & & & 0.0034 & & & 0.0044 & & \\
\hline$T_{\text {or }}$ (HJD) & 2449803.6420 & & & & & & & & & & & \\
\hline
\end{tabular}

This is also suggested from the phase shift derived from $(b-y)$ and $y: \phi_{b-y}-\phi_{y}=-19.1 \pm 12.7$.

Interesting is also the behaviour of HD 129231 concerning the amplitude ratios relative to the filter $u$ and $f_{1}$. It is shown that the amplitude in this filter is the largest in all three datasets. This feature is not typical in $\delta$ Sct-type pulsators (Garrido et al. 1990; Garrido 2000) but rather in hotter pulsating stars such as $\beta$ Cep
(Heynderickx et al. 1994) or SPB (North \& Paltani 1994; Waelkens 1996) variables. However, this peculiarity is also observed in some modes of another $\delta$ Sct star, $\theta$ Tuc (Paparó \& Sterken 2000), being suggested to be connected with the larger frequencies, that is, the higher radial orders $(n)$ of pulsation. This might be the case of HD 129231 where $f_{1}$ is the largest frequency and a value of $0.018 \mathrm{~d}$ can be obtained for the pulsation constant (see Table 8). 


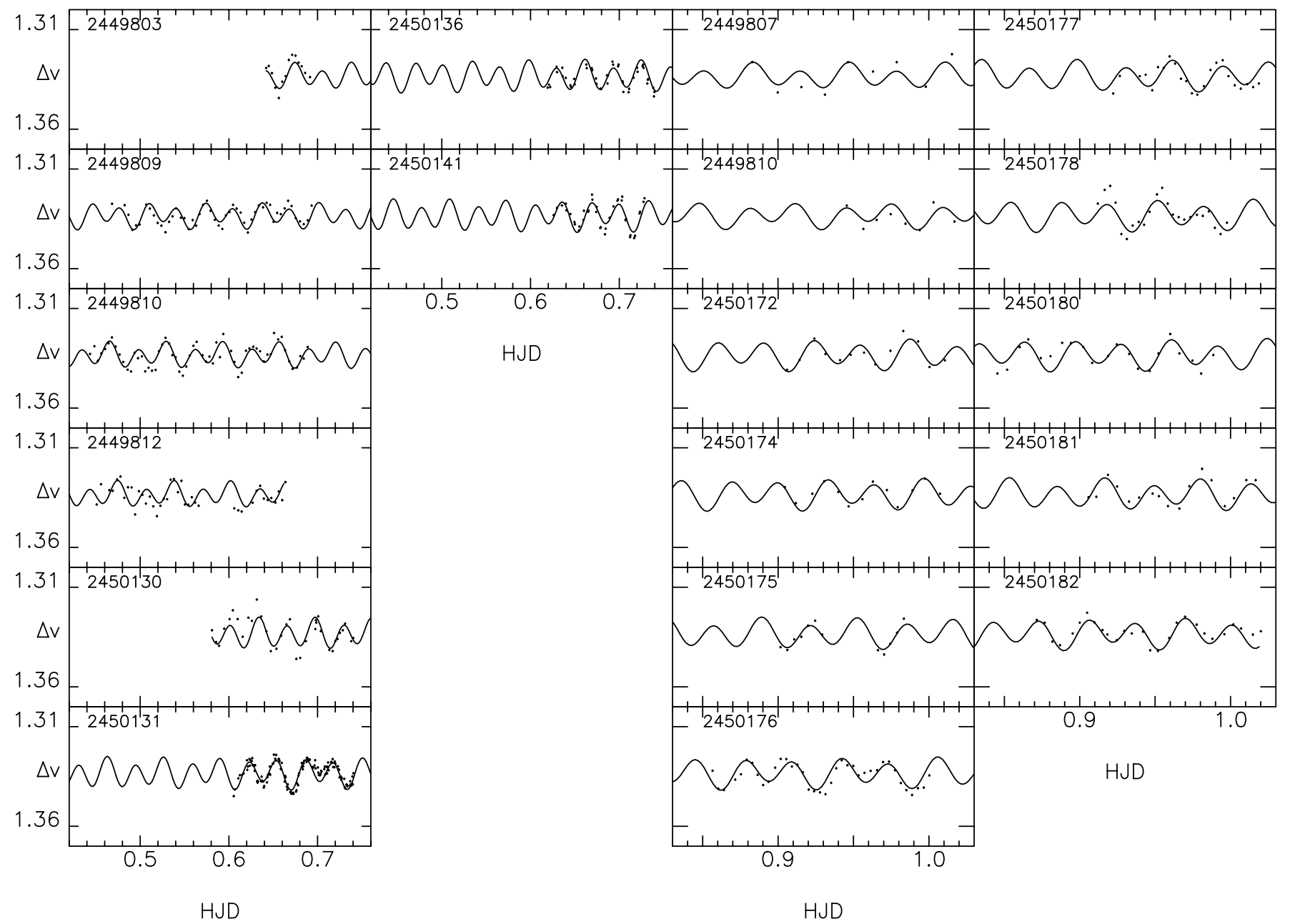

Fig. 3. Same as Fig. 2 for the 1995 and 1996 datasets.

However, it is not occurring in other $\delta$ Sct-type variables with low $Q$ values (e.g., 28 And or AN Lyn; Rodríguez et al. $1993,1997 \mathrm{~b})$.

\section{Photometry}

An estimate of the stellar physical parameters of HD 129231, including absolute magnitude, surface gravity, effective temperature and other quantities can be derived by applying suitable calibrations for $u v b y \beta$ photometry. Using the observed colour indices obtained in the present work (Table 2), we can deredden them making use of Crawford's (1979) calibration for A stars. This way, we derive a nearly null colour excess $\left(E(b-y)=0{ }^{\mathrm{m}} 008\right)$ and the corresponding intrinsic indices of: $(b-y)_{0}=0{ }^{\mathrm{m}} 054$, $m_{0}=0.185$ and $c_{0}=1{ }^{\mathrm{m}} 005$. Furthermore, deviations from the ZAMS's values of $\delta m_{1}=0$. 015 and $\delta c_{1}=0$. 068 are also found. These values suggest this variable being a normal Population I $\delta$ Sct-type pulsating star with solar abundances lying close to the blue border of the $\delta$ Sct instability strip, as it can be seen from Figs. 7 and 8 of Rodríguez \& Breger (2001). In fact, a value of $[\mathrm{Me} / \mathrm{H}]=-0.08$ can be derived using Smalley's (1993) calibration for metal abundances in A stars.
Moreover, using Crawford's (1979) relation for luminosity, we can obtain $M_{v}(\mathrm{ph})=1.64$ with an errorbar of about 0.3 which is commonly assumed for photometry (Rodríguez \& Breger 2001). On the other hand, a larger value of $M_{v}(\pi)=2.36 \pm 0.27$ is obtained using the parallax of $\pi=8.15 \pm 1.00$ mas derived for HD 129231 by the Hipparcos satellite (ESA 1997). Adopting the same procedure that in Rodríguez \& Breger (2001), an average absolute magnitude can be calculated with weighting according to the squares of the corresponding standard deviations. Thus, a value of $2{ }^{\mathrm{m}} 04 \pm 0.28$ can be assumed for $M_{v}$. Furthermore, using the relations by Code et al. (1976) for bolometric correction, Crawford \& Mandwewala (1976) for interstellar reddening and the grids of Smalley \& Kupka (1997) with $[\mathrm{Me} / \mathrm{H}]=0.0$ for calibrations in temperature and gravity, we can obtain the following values of $M_{\text {bol }}=2.02 \pm 0.3$, a distance modulus D.M. $=5.8 \pm 0.3$, $T_{\text {eff }}=8550 \pm 150 \mathrm{~K}$ and $\log g=4.15 \pm 0.1$. Thus, a radius of $1.60 \pm 0.3 R_{\odot}$ can be calculated using the radiation law

$\log R / R_{\odot}=-0.2 M_{\mathrm{bol}}-2 \log T_{\mathrm{eff}}+8.472$.

Moreover, it is possible to gain some insight into the mass and age of this star using the evolutionary tracks for solar abundances by Claret (1995). In this case, values of 
Table 7. Reddening and derived physical parameters for HD 129231.

\begin{tabular}{lclc}
\hline Parameter & Value & Parameter & Value \\
\hline$E(b-y)$ & $0.008 \pm 0.01$ & $M_{\text {bol }}$ & $2^{\mathrm{m}} 02 \pm 0.3$ \\
$(b-y)_{0}$ & $0.054 \pm 0.01$ & D.M. & $5.8 \pm 0.3$ \\
$m_{0}$ & $0.185 \pm 0.01$ & $\log L / L_{\odot}($ dex $)$ & $1.1 \pm 0.1$ \\
$c_{0}$ & $1^{\mathrm{m}} 005 \pm 0.01$ & $T_{\mathrm{e}}(\mathrm{K})$ & $8550 \pm 150$ \\
$\delta m_{1}$ & $0.015 \pm 0.01$ & $\log g(\mathrm{dex})$ & $4.15 \pm 0.1$ \\
$\delta c_{1}$ & $0.068 \pm 0.02$ & Age $(\mathrm{Gyr})$ & $0.1 \pm 0.1$ \\
{$[\mathrm{Me} / \mathrm{H}](\mathrm{dex})$} & $-0.08 \pm 0.1$ & $M / M_{\odot}$ & $1.91 \pm 0.1$ \\
$M_{v}$ & $2^{\mathrm{m}} 04 \pm 0.3$ & $R / R_{\odot}$ & $1.60 \pm 0.3$ \\
\hline
\end{tabular}

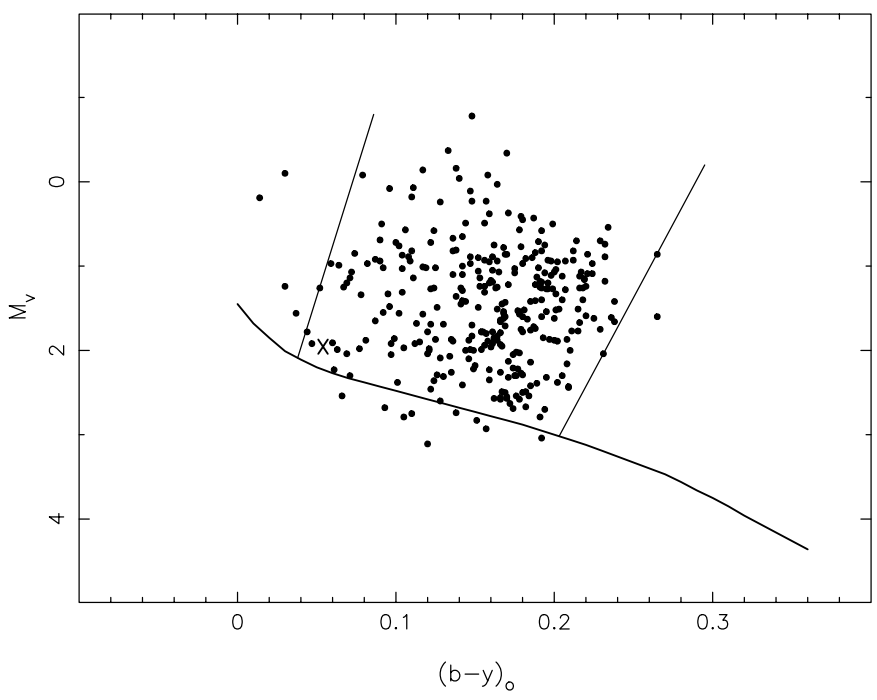

Fig. 4. $\delta$ Sct-type pulsators in the H-R diagram. HD 129231 is shown with the simbol X.

$M=1.91 \pm 0.1 M_{\odot}$ and Age $=0.1 \pm 0.1$ Gyr are found Table 7 summarizes the physical parameters obtained for HD 129231 whereas Fig. 4 shows the position of this variable in the $\mathrm{H}-\mathrm{R}$ diagram together with the full sample of $\delta$ Sct-type pulsators from Rodríguez \& Breger (2001).

Additionally, these results were checked using other routines for calibration and a very good agreement was found with the results obtained above. The modelatmosphere calibrations of Moon \& Dworetsky (1985) were used in order to derive the temperature and gravity of the variable, leading to $T_{\text {eff }}=8420 \mathrm{~K}$ and $\log g=4.10$. The luminosity calibrations of Domingo \& Figueras (1999) were also used leading to $M_{v}=1 \mathrm{~m} 66$, which compares quite well with that of $1 . \mathrm{m} 64$ previously obtained from photometry. This suggests that the $M_{v}(\pi)$ value obtained from the parallax might be overestimated in spite of the relatively small errorbar $\left(\sigma_{M_{v}(\pi)}=0.27\right)$, similar to the discrepancies observed in other $\delta$ Sct variables as, e.g. AD CMi (Høg \& Petersen 1997; Antonello \& Mantegazza 1997; Petersen \& Høg 1998). Its very low luminosity, as inferred from the Hipparcos parallax, could be explained by the systematic effects related to observational errors.

On the other hand, the results on stellar mass, radius and surface gravity were also checked against those derived using the biparametric calibrations of Ribas et al. (1997)
Table 8. Pulsation constants for the frequencies detected in HD 129231.

\begin{tabular}{ccc}
\hline Freq. $\left(\mathrm{cd}^{-1}\right)$ & Freq. $(\mu \mathrm{Hz})$ & $Q(d)$ \\
\hline$f_{1}=31.355$ & 362.91 & 0.018 \\
$f_{2}=16.923$ & 195.87 & 0.033 \\
$f_{3}=17.130$ & 198.26 & 0.033 \\
$f_{4}=17.067$ & 197.53 & 0.033 \\
$f_{5}=22.260$ & 257.64 & 0.025 \\
\hline
\end{tabular}

with $u v b y \beta$ photometry. Thus, the following values can be obtained $M=1.90 M_{\odot}, R=1.73 R_{\odot}$ and $\log g=4.24$, in very good agreement with those listed in Table 7.

The pulsation constants $Q_{i}$ corresponding to each of the frequencies detected in HD 129231 can be calculated using the empirical formula derived by Petersen \& Jørgensen (1972). The results are listed in Table 8. From this table it is evident that nonradial modes are present in the pulsation of this variable. Two, at least, of the three frequencies $f_{2}, f_{3}$ and $f_{4}$ correspond to nonradial modes. Indeed, these frequencies might result from rotational splitting with the same $n$ and $l$ numbers (Saio 1981). Moreover, $f_{1}$ was suggested in the previous section to be a nonradial mode too. According to the derived $Q$ values, the frequencies detected for this variable obey to three different families of radial orders $(n=0,1$ and 3$)$ (Fitch 1981). Nevertheless, the mode identification for the detected frequencies is premature and obviously this variable deserves further study.

\section{Conclusions}

In this work we present the results of photometric observations carried out during three consecutive years (1995 to 1997$)$ on the recently discovered $\delta$ Sct-type variable HD 129231. The observations consist of simultaneous uvby measurements together with a few data obtained in the $\mathrm{H}_{\beta}$-Crawford system. These observations were collected at two observatories (Sierra Nevada, Spain and San Pedro Mártir, Mexico) using identical instrumentation.

The frequency content has been analysed through the three years leading to the following results:

(i) Multiperiodic behaviour is found with each of the datasets. A set of five significant frequencies is detected as the best fitting for the 1997 dataset and an additional frequency $f_{6}=f_{3}+f_{4}$ is probably also present in these data. However, only the two main frequencies $f_{1}$ and $f_{2}$ are found as being significant in the 1995 and 1996 observing runs. This could be due, at least in part, to the poorer quality of these data. We expect that further observations on this variable lead to a better resolution of the pulsational content and behaviour.

(ii) Nevertheless, amplitude variations are suggested in this variable for the frequencies $f_{3}$ and later. This is also true for $f_{1}$, but no variations are found for $f_{2}$ within the observational errorbars.

(iii) The pulsational amplitude in the $u$ band is larger than in all the other three bands, including the $v$ filter, for 
the frequency $f_{1}$. This behaviour is not common among the $\delta$ Sct-type pulsators.

(iv) The pulsation constants corresponding to each detected frequency were calculated, but a mode identification is presently premature. Nonradial pulsation is suggested for, at least, 3 modes including that corresponding to $f_{1}$. The nonradial nature of $f_{1}$ is suggested from the observed phase shifts between different filters. Futhermore, rotational splitting might also exist as related to $f_{2}, f_{3}$ and $f_{4}$.

The physical parameters of HD 129231 were determined using the uvby $\beta$ indices derived in this work together with suitable Strömgren photometric calibrations available in the bibliography, including atmosphere and stellar evolution models. The results indicate this variable to be a normal Population I $\delta$ Sct-type pulsator with solar abundances located close to the blue edge of the $\delta$ Sctinstability region evolving on its main sequence stage.

Acknowledgements. This research was supported by the Junta de Andalucía and the Dirección General de Investigación (DGI) under project AYA2000-1559. This research has made use of the Simbad database, operated at CDS, Strasbourg, France.

\section{References}

Antonello, E., \& Mantegazza, L. 1997, A\&A, 327, 240

Breger, M. 1990, Comm. Asteroseismol., 20, 1

Breger, M., Stich, J., Garrido, R., et al. 1993, A\&A, 271, 482

Breger, M., Handler, G., Garrido, R., et al. 1999, A\&A, 349,225

Claret, A. 1995, A\&AS, 109, 441

Code, A. D., Davis, J., Bless, R. C., \& Brown, R. H. 1976, ApJ, 203, 417

Crawford, D. L. 1979, AJ, 84, 1858

Crawford, D. L., \& Mandwewala, N. 1976, PASP, 88, 917

Deeming, T. J. 1975, Ap\&SS, 36, 137

Domingo, A., \& Figueras, F. 1999, A\&A, 343, 446

ESA 1997, The Hipparcos and Tycho Catalogues, ESA SP-1200

Fitch, W. S. 1981, ApJ, 249, 218
Garrido, R. 2000, PASPC, 210, 67

Garrido, R., García-Lobo, E., \& Rodríguez, E. 1990, A\&A, 234,262

Hauck, B., \& Mermilliod, M. 1998, A\&AS, 129, 431

Heynderickx, D., Waelkens, C., \& Smeyers, P. 1994, A\&AS, 105, 447

Høg, E., \& Petersen, J. O. 1997, A\&A, 323, 827

Montgomery, M. H., \& O'Donoghue, D. 1999, Delta Scuti Star Newsletter, 13, 28

Moon, T. T., \& Dworetsky, M. M. 1985, MNRAS, 217, 305

Nielsen, R. F. 1983, Inst. Theor. Astrophys. Oslo Report, No. 59, ed. O. Hauge, 141

North, P., \& Paltani, S. 1994, A\&A, 288, 155

Paparó, M., \& Sterken, C. 2000, A\&A, 362, 245

Penprase, B. E. 1992, ApJS, 83, 273

Petersen, J. O., \& Høg, E. 1998, A\&A, 331, 989

Petersen, J. O., \& Jørgensen, H. E. 1972, A\&A, 17, 367

Ribas, I., Jordi, C., Torra, J., \& Giménez, A. 1997, A\&A, 327, 207

Rodríguez, E., \& Breger, M. 2001, A\&A, 366, 178

Rodríguez, E., Rolland, A., López de Coca, P., Garrido, R., \& Mendoza, E. E. 1993, A\&A, 273, 473

Rodríguez, E., González-Bedolla, S. F., Rolland, A., Costa, V., \& López de Coca, P. 1997a, A\&A, 324, 959

Rodríguez, E., González-Bedolla, S. F., Rolland, A., et al. 1997b, A\&A, 328, 235

Rodríguez, E., Rolland, A., López-González, M. J., \& Costa, V. 1998, A\&A, 338, 905

Rodríguez, E., González-Bedolla, S. F., López-González, M. J., Rolland, A., \& Costa, V. 1999a, Inf. Bull. Var. Stars, No. 4686

Rodríguez, E., González-Bedolla, S. F., López-González, M. J., Rolland, A., \& Costa, V. 1999b, Inf. Bull. Var. Stars, No. 4694

Rodríguez, E., López-González, M. J., \& López de Coca, P. 2000, A\&AS, 144, 469

Saio, H. 1981, ApJ, 244, 299

Simbad 2001, Simbad Database, CDS, Strasbourg, France

Smalley, B. 1993, A\&A, 274, 391

Smalley, B., \& Kupka, F. 1997, A\&A, 328, 349

Sperl, M. 1998, Comm. Asteroseismol., 111, 1

Waelkens, C. 1996, A\&A, 311, 873 